\title{
Effect of procalcitonin on the prognosis of patients with COPD
}

\author{
CHEN GONG ${ }^{*}$, YING YANG ${ }^{*}$, MINLI CHEN and ZHENGFU XIE \\ Geriatrics Respiratory Medicine, The First Affiliated Hospital of Guangxi Medical University, \\ Nanning, Guangxi 530021, P.R. China
}

Received September 25, 2019; Accepted March 16, 2020

DOI: $10.3892 /$ br.2020.1298

\begin{abstract}
The aim of the present study was to investigate the effect of procalcitonin levels on the prognosis of chronic obstructive pulmonary disease (COPD), and its relationship with other indices of infection. Inpatients diagnosed with acute aggravation of COPD between January 2017 and June 2018 were enrolled in the present study. Troponin, C-reactive protein (CRP), erythrocyte sedimentation rate (ESR), white blood cell (WBC) count, percentage of neutrophils (NE\%), hospitalization days and hospitalization expenses were collected and followed up for half a year to observe the survival rate in patients with COPD and the risk of acute aggravation. There were 246 patients with procalcitonin (PCT) levels $<0.1 \mathrm{ng} / \mathrm{ml}$, among whom 55 were randomly selected and assigned to a PCT negative group; and another 55 patients with PCT levels $\geq 0.1 \mathrm{ng} / \mathrm{ml}$ were assigned to a PCT positive group. The serum CRP, ESR, WBC count, NE\% and hospitalization expenses in the PCT positive group were significantly higher compared with the PCT negative group $(\mathrm{P}<0.05)$. There was a positive association between PCT levels and CRP, WBC, NE\% and hospitalization expenses, but not between PCT and ESR. The number of AECOPD events occurring within half a year between the two groups was statistically significant $\left(\chi^{2}=5.923\right.$; $\mathrm{P}<0.05$ ), and there was no significant correlation between PCT and recurrence frequency. Together, the results of the present study suggest that the levels of PCT in patients with acute aggravation may reflect the severity of COPD and may be used as a reference value for prognostic risk assessment. Serum PCT levels may be used as an indicator to predict duration and cost of hospitalization.
\end{abstract}

Correspondence to: Dr Minli Chen, Geriatrics Respiratory Medicine, The First Affiliated Hospital of Guangxi Medical University, 6 Shuangyong Road, Nanning, Guangxi 530021, P.R. China

E-mail:nncml@163.com

*Contributed equally

Key words: chronic obstructive pulmonary disease, procalcitonin, white blood cell count, neutrophil percentage, C-reactive protein, erythrocyte sedimentation rate

\section{Introduction}

Repeated acute episodes in patients with chronic obstructive pulmonary disease (COPD) is a primary cause of repeated hospitalization and death. The mortality rate in patients with COPD is positively associated with the number of hospitalizations caused by acute exacerbations (1). The frequency and severity of acute exacerbation of COPD (AECOPD) events are vital for predicting the prognosis of patients with COPD (2). It was previously shown that non-infectious factors cause $20 \%$ of AECOPD cases, and most acute exacerbations of COPD are caused by infection (3). Fever, pyoptomia, increased cough frequency and aggravation of dyspnea are common clinical symptoms in patients with COPD at the acute exacerbation stage. Patients may even require admittance to an intensive care unit due to respiratory failure and require assisted breathing. Acute exacerbations in patients with COPD increases airway inflammation, increases the synthesis of inflammatory factors, activates the complement system, and results in increased inflammation in the body (4).

Increased airway inflammation is frequently observed in AECOPD. As infectious factors are the most critical factors inducing AECOPD, the emergence of AECOPD may result in aggravation of the body's inflammatory response, which would inevitably result in changes to indicators of infection in blood circulation (5). This infectious inflammatory standard is essential for the diagnosis and treatment of AECOPD (6). For several years, researches have been looking for markers of infectious inflammation in the blood during acute exacerbations of COPD. Thus far, several related markers have been identified, including erythrocyte sedimentation rate (ESR), C-reactive protein (CRP), white blood cell (WBC) count and neutrophil percentage (NE\%), which are commonly used in determining the acute exacerbations and severity of COPD in patients during hospitalization $(7,8)$. Other, less conventional markers include triggering receptor expressed on myeloid cells- 1 , tumor necrosis factor receptor- $\alpha$, interleukin (IL)- 8 and IL-6 and matrix metalloproteinases (MMPs) (9); yet, these markers are not widely used clinically, which may be due to the complexity of detection methods.

Previously, a novel infectious marker, procalcitonin (PCT), has been used as an indicator of infection during AECOPD. PCT and other infectious inflammatory factors are associated with the progression of COPD; but there are relatively fewer studies on the prognostic value of PCT in COPD (10). 
Repeated AECOPD events and the number of hospitalizations are important factors associated with the increase of mortality in patients with COPD. Therefore, the aim of the present study was to determine whether the PCT levels at admission in patients with AECOPD was associated with the number of hospitalizations of patients due to acute exacerbations following the initial discharge; and whether the prognosis of patients with COPD could be predicted by monitoring PCT levels. Briefly, patients with different levels of PCT were followed up by telephone for 6 months and relevant outpatient medical records were used to investigate whether PCT levels were a useful metric for predicting the prognosis of patients with COPD. WBC, NE\%, CRP and ESR values detected on admission were collected, and analyzed to determine whether there was an association between PCT and these traditional inflammatory markers of infection. Additionally, the number of days of hospitalization, the cost of hospitalization and the levels of PCT were analyzed to assess whether the levels of PCT were associated with the length of stay and the cost of hospitalization.

\section{Patients and methods}

Patients. A total of 110 patients who were admitted to the First Affiliated Hospital of Guangxi Medical University for AECOPD between January 2017 and June 2018 were enrolled. The inclusion criteria were as follows: i) Patient or legal guardian provided signed informed consent; ii) patient diagnosed according to diagnostic criteria of the global initiative for chronic obstructive lung disease guidelines and graded as D (11); and iii) patient tested for PCT by blood sampling within $24 \mathrm{~h}$ of admission. Patients with a tumor, bronchial asthma, tuberculosis, severe liver or renal insufficiency, or had any other sites with definite infection were excluded.

Data on 792 patients who experienced AECOPD was collected. Among these, there were 391 patients who were not tested for PCT within $24 \mathrm{~h}$ of admission (PCT examination was not included in the medical insurance, and/or patients/patients' guardian refused the test), 36 patients with complicated tumors, 25 patients with severe liver and renal insufficiency, 11 patients with bronchiectasis and infection, 9 patients with bronchial asthma, 8 patients with tuberculosis, 6 patients with urinary tract infection and 5 patients with ringomycosis, all of whom were excluded from the present study. In the positive group, serum PCT levels $\geq 0.1 \mathrm{ng} / \mathrm{ml}$ were observed in 55 patients, including 51 males and 4 females. The mean age \pm standard deviation was $72.49 \pm 10.57$ years. The serum PCT in the 55 patients from the negative group was $<0.1 \mathrm{ng} / \mathrm{ml}$; there were 52 males and 3 females in this group, and the mean age was $71.74 \pm 9.29$ years old (Table I).

All patient data were obtained from the medical record information management system (DT Health 6, Donghua digital hospital information management system) of the First Affiliated Hospital of Guangxi Medical University. Patients were divided into groups according to the levels of PCT; PCT $>0.1 \mathrm{ng} / \mathrm{ml}$, positive group and $\mathrm{PCT}<0.1 \mathrm{ng} / \mathrm{ml}$, negative group.

Data on PCT, ESR, CRP, WBC counts, and NE\%, hospitalization expense and hospitalization days were collected and sorted through the auxiliary examinations during hospitalization of a total of 110 patients in the two groups. At the same
Table I. Age and sex distribution among the PCT positive and negative groups.

\begin{tabular}{lccccc}
\hline & \multicolumn{2}{c}{ PCT positive } & & \multicolumn{2}{c}{ PCT negative } \\
\cline { 2 - 3 } \cline { 5 - 6 } Age & No. of cases & Sex (M/F) & & No. of cases & Sex (M/F) \\
\hline $50-59$ & 7 & $7 / 0$ & & 6 & $6 / 0$ \\
$60-69$ & 13 & $11 / 2$ & & 12 & $12 / 0$ \\
$70-79$ & 20 & $20 / 2$ & & 23 & $23 / 0$ \\
$80-89$ & 13 & $13 / 0$ & & 14 & $11 / 3$ \\
$>90$ & 2 & $2 / 0$ & & - & - \\
Total & 55 & $51 / 4$ & & 55 & $52 / 3$ \\
\hline
\end{tabular}

PCT, procalcitonin; M, male; F, female.

Table II. Compliance during remission in the PCT positive and negative groups.

\begin{tabular}{lccc}
\hline Group & $\begin{array}{c}\text { Standardized } \\
\text { medication }\end{array}$ & $\begin{array}{c}\text { Nonstandard } \\
\text { medication }\end{array}$ & Total \\
\hline PCT positive & 35 & 16 & 51 \\
PCT negative & 33 & 18 & 51 \\
\hline
\end{tabular}

PCT, procalcitonin.

time, these patients were followed up by telephone every month for 6 months, including reviewing the patient's medical records to obtain the relevant patients' information. The frequency of reoccurrence of AECOPD within half a year and the outcomes after discharge were observed. December 25, 2018 was set as the final observation date.

Statistical analysis. The obtained data were statistically processed by SPSS version 23 (IBM Corp.). The measurement data were tested for normality and homogeneity of variance. The measurement data conforming to normal distribution are expressed as the mean \pm standard deviation, and the difference between groups was assessed using a Student's t-test. The measurement data with skewed distribution are expressed as median and the interquartile range. If the correlation analysis between the two variables satisfied the linear correlation, Pearson linear correlation was used; if not, Spearman' rank correlation analysis was used. The number of AECOPD events occurring within half a year between the two groups was analyzed using a $\chi^{2}$ test. $\mathrm{P}<0.05$ was considered to indicate a statistically significant difference.

\section{Results}

Patient characteristics. After screening for the inclusion and exclusion criteria, 301 patients met the criteria. There were 55 patients with PCT $\geq 0.1 \mathrm{ng} / \mathrm{ml}$ and 246 patients with PCT $<0.1 \mathrm{ng} / \mathrm{ml}$. The 55 cases with PCT $\geq 0.1 \mathrm{ng} / \mathrm{ml}$ were used as the PCT positive group, and 55 of the 246 individuals with $\mathrm{PCT}<0.1 \mathrm{ng} / \mathrm{ml}$ were randomly selected for inclusion in the 
Table III. Comparison of levels of serum inflammatory factors between the PCT positive and negative groups (mean \pm SD).

\begin{tabular}{lccrr}
\hline Factor & PCT positive & PCT negative & t & P-value \\
\hline CRP, $\mathrm{mg} / \mathrm{l}$ & $50.86 \pm 33.49$ & $18.21 \pm 13.90$ & 6.677 & $<0.05$ \\
ESR, mm/h & $40.38 \pm 19.89$ & $17.28 \pm 12.37$ & 7.312 & $<0.05$ \\
WBC count, $10^{9} / 1$ & $11.05 \pm 6.24$ & $8.50 \pm 3.37$ & 2.624 & $<0.05$ \\
NE\% & $0.78 \pm 0.11$ & $0.71 \pm 0.12$ & 3.177 & $<0.05$ \\
\hline
\end{tabular}

PCT, procalcitonin; CRP, C-reactive protein; ESR, erythrocyte sedimentation rate; WBC, white blood cell; NE\%, percentage of neutrophils.

Table IV. Correlation analysis between PCT and other inflammatory factors.

\begin{tabular}{lcc}
\hline Factor & $\mathrm{R}$ & P-value \\
\hline CRP & 0.201 & $<0.05$ \\
ESR & 0.150 & $>0.05$ \\
WBC & 0.512 & $<0.05$ \\
NE\% & 0.212 & $<0.05$ \\
\hline
\end{tabular}

PCT, procalcitonin; CRP, C-reactive protein; ESR, erythrocyte sedimentation rate; $\mathrm{WBC}$, white blood cell; NE\%, percentage of neutrophils.

Table V. Comparison of hospital expenses between the PCT positive and negative groups.

\begin{tabular}{lcc}
\hline Groups & No. of cases & $\begin{array}{c}\text { Hospitalization } \\
\text { expenses, } \text { RMB }^{\mathrm{a}}\end{array}$ \\
\hline PCT positive & 55 & $26,666.04 \pm 21,702.25$ \\
PCT negative & 55 & $19,034.96 \pm 11,740.26$ \\
\hline
\end{tabular}

${ }^{a} \mathrm{t}=3.146, \mathrm{P}<0.05$. PCT, procalcitonin; RMB, Chinese Yuan.

Table VI. Follow-up of the patients in the PCT positive and negative group.

\begin{tabular}{lcc}
\hline $\begin{array}{l}\text { Follow-up } \\
\text { investigation }\end{array}$ & $\begin{array}{c}\text { PCT positive, } \\
\mathrm{n}(\%)\end{array}$ & $\begin{array}{c}\text { PCT negative, } \\
\mathrm{n}(\%)\end{array}$ \\
\hline $\begin{array}{l}\text { Acute exacerbation } \\
\text { Stable phase }\end{array}$ & $26(47.2)$ & $14(25.4)$ \\
Loss to follow-up & $25(45.5)$ & $37(67.2)$ \\
Total & $4(7.3)$ & $4(7.3)$ \\
\end{tabular}

PCT, procalcitonin.

PCT negative group. There were no statistically significant differences in sex and age between the two groups $(\mathrm{P}>0.05)$. In the PCT-positive group, 45 patients (44 men and 1 woman) had a history of long-term smoking, and two patients had a history of long-term dust exposure. In the PCT negative group, 50 patients had a history of long-term smoking, 2 female patients and 1 male patient had a history of long-term dust exposure. In the PCT positive group, there were 11 patients with hypertension (20\%), 1 patient with type 2 diabetes $(1.8 \%)$, 3 patients with coronary heart disease $(5.4 \%), 4$ patients with $\geq 2$ diseases $(7.0 \%)$, and 11 patients with other diseases (20\%). In the PCT-negative group, 12 patients $(22.0 \%)$ were associated with hypertension, 2 patients $(3.6 \%)$ with type 2 diabetes, 2 patients (3.6\%) with coronary heart disease, 4 patients $(7.0 \%)$ with $\geq 2$ diseases and 14 patients $(36 \%)$ with other diseases. Patients in both groups were discharged with inhaled corticosteroids + long-acting $\beta$ agonist + long acting anticholinergic agents, without glucocorticoids or antibiotics. After discharge, 35 patients in the PCT positive group received regular medication during the COPD stable period, and 16 patients received irregular medication. After discharge, 33 patients in the PCT negative group received regular medication during the COPD stable period, and 18 patients received irregular medication (Table II).

Comparison of other inflammatory indicators between the PCT positive group and the PCT negative group. In the PCT positive group, the CRP levels were $50.86 \pm 33.49 \mathrm{mg} / \mathrm{l}$, whereas in the PCT negative group they were $18.21 \pm 13.90 \mathrm{mg} / \mathrm{l}, \mathrm{t}$ $=6.677$. This difference was statistically significant $(\mathrm{P}<0.05)$. In the PCT positive group, ESR fluctuated at $40.38 \pm 19.89 \mathrm{~mm} / \mathrm{h}$, whereas in the PCT negative group, it fluctuated at $17.28 \pm 12.37 \mathrm{~mm} / \mathrm{h}, \mathrm{t}=7.312(\mathrm{P}<0.05)$. The WBC count in the PCT positive group was $11.05 \pm 6.24 \times 10^{9} / 1$, whereas in the PCT negative group it was $8.50 \pm 3.37 \times 10^{9} / 1, \mathrm{t}=2.624(\mathrm{P}<0.05)$. In the PCT positive group, NE\% was $0.78 \pm 0.11$, and in the PCT negative group it was $0.71 \pm 0.12, \mathrm{t}=3.177(\mathrm{P}<0.05)$. The serum levels of CRP, ESR, WBC and NE\% in the PCT positive group were all higher compared with the PCT negative group (Table III, Fig. S1).

Correlation between PCT with CRP, ESR, WBC and NE\% in patients with acute exacerbations of COPD. PCT, CRP, ESR, WBC and NE\% of the 110 patients were analyzed. The results of Pearson correlation analysis were as follows: PCT was positively correlated with $\mathrm{CRP}(\mathrm{R}=0.301)$. There was no significant correlation between PCT and ESR. PCT was positively correlated with WBC count $(\mathrm{R}=0.512)$, with $\mathrm{NE} \%,(\mathrm{R}=0.212)$. Furthermore, PCT was positively correlated with CRP, WBC and NE\% in patients with AECOPD, and PCT showed positive association with the increase of the aforementioned indicators. 
There was no significant correlation between PCT and ESR (Table IV, Fig. S2).

Comparison of hospitalization expenses between the PCT positive and PCT negative groups. The inpatient expenses of the PCT positive group were $26,666.04 \pm 21,702.25$ yuan, and the inpatient expenses of the PCT negative group were $19,034.96 \pm 11,740.26$-yuan, $t=3.146$, and the difference was significant $(\mathrm{P}<0.05)$. These results suggest that the hospitalization costs of patients in the PCT positive group were higher compared with the PCT negative group.

Pearson correlation analysis was performed to analyze the hospitalization expenses of the two groups. The results suggested that PCT was positively correlated with hospitalization expenses and that the hospitalization expenses increased in-line with an increase in PCT levels $(\mathrm{R}=0.315, \mathrm{P}<0.05)$.

Spearman correlation analysis was performed on the number of days in hospital and PCT levels in the two groups, and the results suggested that PCT was positively correlated with the number of days spent at hospital in the patients with acute onset of $\operatorname{COPD}(\mathrm{R}=0.312, \mathrm{P}<0.05)$, and the number of days at hospital increased with an increase in PCT level (Table V).

Follow-up results of patients in the two groups. After the discharge of AECOPD patients from both groups, each patient was followed up by telephone for 6 months to observe the 6-month survival rate in both groups and the risk of recurrence of AECOPD events after discharge. In the PCT-positive group, 8 patients died within 6 months after discharge, including one death due to cerebral infarction, 1 sudden death due to unknown cause, and six deaths due to acute exacerbation of COPD. In the PCT positive group, 2 patients died due to acute exacerbations of COPD with one acute exacerbation, 3 patients died due to two acute exacerbations, and 1 patient died due to four acute exacerbations. Of the remaining 47 patients, 2 were lost to follow-up, and 20 of the remaining 45 patients were hospitalized with acute exacerbations of COPD, including 13 patients with one acute attack, 3 patients with two acute exacerbations, 3 patients with three acute exacerbations, and 1 patient with six acute exacerbations. In the PCT-negative group, 4 patients died within 6 months of discharge; 3 patients died from acute exacerbations of COPD, and 1 from other causes. All patients in the PCT negative group died from acute exacerbation of COPD with one acute exacerbation. Additionally, 3 of the remaining 51 patients were lost to follow-up, and 11 of the remaining 48 patients were hospitalized with acute exacerbations of COPD within half a year, where 7 patients had one acute exacerbation of COPD and 4 patients had two acute exacerbations. The number of AECOPD events occurring within half a year between the two groups was analyzed by SPSS 23.0 software using a $\chi^{2}$ test. The difference between the two groups was statistically significant $\left(\chi^{2}=5.923, \mathrm{P}<0.05\right)$, indicating that the risk of AECOPD events in the PCT positive group within 6 months was significantly higher compared with the PCT negative group (Table VI).

\section{Discussion}

PCT is a non-hormone-active protein composed of 116 amino acids, with a molecular weight of $\sim 13,000 \mathrm{kDa}$ and was first discovered in 1992 (12). The production of PCT is rather complex. PCT is primarily generated by calcitonin cleavage in thyroid C cells, whereas some PCT may be synthesized by other cells. For example, certain patients with small-cell lung cancer have increased blood PCT levels, suggesting that specific neuroendocrine cells in the lungs may synthetize PCT (13). Physiologically, PCT is primarily produced by thyroid C cells; however, when the body is infected, PCT may be produced by other organs as well (14). PCT levels are also significantly increased in sepsis patients with thyroid resection (15). PCT levels in healthy adults are low, generally $<0.1 \mathrm{ng} / \mathrm{ml}$; however, PCT has high sensitivity and specificity, and levels are significantly increased in the presence of bacterial infection, sepsis and other conditions, and may increase by $>1,000$ times the baseline value (15). PCT can be detected within 2-4 h of infection and peaks within 6-24 h. Its half-life in blood circulation is 25-30 $\mathrm{h}$. The only available pathway for removal is renal metabolism, and the metabolism of PCT is slightly prolonged in patients with renal dysfunction (16). PCT is not affected by drugs such as glucocorticoids and non-steroidal drugs, and is not affected by the body's own immune response, and thus, the levels do not significantly increase in patients with viral infections (17). At present, PCT has been widely used to guide the use of antibiotics in clinical practice, and may also be used to predict the prognosis of certain diseases (18).

In the present study, 110 patients with COPD were divided into a PCT positive and PCT negative group. Analysis showed the mortality rate and readmission due to AECOPD events in the PCT positive group were higher compared with the PCT negative group. These results highlight the potential predictive value of PCT levels in patients hospitalized with AECOPD, and may be used to guide prognosis following discharge. Patients with high PCT levels are at an increased risk of re-exacerbation due to COPD compared with those with relatively lower levels of PCT. It has been reported that the PCT levels may be useful for predicting the mortality and prognosis of community-acquired pneumonia (CAP), and the PCT levels at admission may be used to predict the severity, prognosis and risk of death due to CAP (17). Most acute exacerbations of COPD are caused by infection, particularly respiratory tract infection, whereas CAP is a relatively common disease. Infection may be associated with the significance of PCT in the prognosis of patients with COPD, and thus requires further study to verify this hypothesis. The number of days of hospitalization and hospitalization costs of patients with higher levels of PCT and AECOPD were analyzed, and there was a significant correlation between patients with higher PCT levels with cost and number of days of hospitalization. Therefore, this indirectly suggests that the patientswith COPD with higher PCT levels exhibited more severe exacerbations.

Commonly used markers of infection used clinically and PCT levels were statistically analyzed in the present study. C-reactive protein is an acute reactive phase protein, and the levels increase in the body within 6-9 h, and has good diagnostic value of infectious diseases (19). The WBC count is significantly increased during infection; but an infection is usually determined by combining the percentage of centrioles (20). ESR is also valuable for the determining activity or quiescence of a disease and for monitoring changes in 
disease condition. Based on the degree of inflammation in the two groups of patients in the present study, the levels of CRP, ESR, WBC and NE\% were higher in the PCT positive group compared with the PCT negative group. Additionally, among all the patients included in the present study, the PCT levels and the levels of inflammatory indicators suggested that the changes in PCT and CRP, WBC, NE\% were positively correlated, although there was no correlation between PCT and ESR. It can be inferred from the above results that PCT, may be a novel inflammatory marker which is correlated with the levels of certain established markers. The correlation coefficients of PCT with WBC was slightly higher, whereas the correlation coefficients with CRP and NE\% were lower, suggesting that PCT is not highly correlated with WBC and NE\%. However, the above results suggest that PCT levels may be affected by certain inflammatory cytokines in the serum.

The present study has some limitations. In the study, there were 110 patients divided into two groups, which is a relatively small sample size. Due to time limitations, the follow-up time was only half a year. Additionally, fewer women were enrolled. Consequently, the results of the present study cannot be generalized to all patients with COPD. Future studies should include larger cohorts and longer follow-up times. They should also account for the influence of season on the incidence of COPD, and include other inflammatory indicators to screen out the most specific indicators for prognosis of COPD. Another limitation of the present study is that patients were followed up with a telephone call every month. Plotting Kaplan-Meir curves plot for exacerbation and death would be beneficial for statistically analyzing exacerbations and mortality. Unfortunately, the time of acute exacerbations and the time of death after discharge were not recorded.

In summary, the PCT levels during AECOPD may reflect the severity of the onset of COPD. Thus, PCT levels may be used as a reference to predict prognosis. Patients with AECOPD with high PCT levels should be more closely monitored and should undergo regular reviews and prescribed drugs following discharge if necessary. PCT levels are also affected by other inflammatory factors in the serum, thus disease assessment and prognostic risk of patients can be evaluated in combination with a number of other established factors.

Frequent exacerbations of COPD have an adverse impact on the long-term survival rate of patients with COPD. Thus, more sensitive and accurate indicators are required for evaluating prognosis.

\section{Acknowledgements}

Not applicable.

\section{Funding}

This study was supported by the Guangxi Health Committee Self-raised Fund (grant no. Z20190798).

\section{Availability of data and materials}

The datasets used and/or analyzed during the present study are available from the corresponding author on reasonable request.

\section{Authors' contributions}

ZX and MC conceived the study, designed the experiments as well as reviewed and edited the manuscript. CG and YY performed the experiments, collected and analyzed the data as well as wrote the manuscript. All authors approved the final version of the manuscript.

\section{Ethics approval and consent to participate}

The present study was approved by the Ethics Committee of The First Affiliated Hospital of Guangxi Medical University (Nanning, China). All patients or legal guardians provided signed informed consent.

\section{Patient consent for publication}

Not applicable.

\section{Competing interests}

The authors declare that they have no competing interests.

\section{References}

1. Flattet Y, Garin N, Serratrice J, Perrier A, Stirnemann J and Carballo S: Determining prognosis in acute exacerbation of COPD. Int J Chron Obstruct Pulmon Dis 12: 467-475, 2017.

2. Dixit D, Bridgeman MB, Andrews LB, Narayanan N, Radbel J, Parikh A and Sunderram J: Acute exacerbations of chronic obstructive pulmonary disease: Diagnosis, management, and prevention in critically ill patients. Pharmacotherapy 35: 631-648, 2015.

3. Sethi S: Infectious etiology of acute exacerbations of chronic bronchitis. Chest 117 (5 Suppl 2): 380S-385S, 2000.

4. Holden V, Slack III D and McCurdy MT: Diagnosis and management of acute exacerbations of chronic obstructive pulmonary disease. Emerg Med Pract 19: 1-24, 2017.

5. Hakamy A, McKeever TM, Steiner MC, Roberts CM, Singh SJ and Bolton CE: The use of the practice walk test in pulmonary rehabilitation program: National COPD audit pulmonary rehabilitation workstream. Int $\mathbf{J}$ Chron Obstruct Pulmon Dis 12: 2681-2686, 2017.

6. Miravitlles M and Anzueto A: Role of infection in exacerbations of chronic obstructive pulmonary disease. Curr Opin Pulm Med 21: 278-283, 2015.

7. Cazzola M, MacNee W, Martinez FJ, Rabe KF, Franciosi LG, Barnes PJ, Brusasco V, Burge PS, Calverley PM, Celli BR, et al: Outcomes for COPD pharmacological trials: From lung function to biomarkers. Eur Respir J 31: 416-469, 2008.

8. Biomarkers Definitions Working Group: Biomarkers and surrogate endpoints: Preferred definitions and conceptual framework. Clin Pharmacol Ther 69: 89-95, 2001.

9. Miller M, Cho JY, Pham A, Ramsdell J and Broide DH: Adiponectin and functional adiponectin receptor 1 are expressed by airway epithelial cells in chronic obstructive pulmonary disease. J Immunol 182: 684-691, 2009.

10. Liu YJ, Du P and Rao J: Procalcitonin as a diagnostic and prognostic marker for sepsis caused by intestinal infection: A case report. Eur Rev Med Pharmacol Sci 17: 1311-1313, 2013.

11. Irusen EM: Deciphering the new global initiative for chronic obstructive lung disease (GOLD) guideline. Curr Respir Care Rep 1: 183-188, 2012.

12. Müller B and Becker KL: Procalcitonin: How a hormone became a marker and mediator of sepsis. Swiss Med Wkly 131: 595-602, 2001.

13. Bohuon C: A brief history of procalcitonin. Intensive Care Med 26 (Suppl 2): S146-S147, 2008.

14. Fisher LA, Kikkawa DO, Rivier JE, Amara SG, Evans RM, Rosenfeld MG, Vale WW and Brown MR: Stimulation of noradrenergic sympathetic outflow by calcitonin gene-related peptide. Nature 305: 534-536, 1983. 
15. Snider RH Jr, Nylen ES and Becker KL: Procalcitonin and its component peptides in systemic inflammation: Immunochemical characterization. J Investig Med 45: 552-560, 1997.

16. Julie D: Procalcitonin. J Clin Pathol 68: 675-679, 2015.

17. Gilbert DN: Procalcitonin as a biomarker in respiratory tract infection. Clin Infect Dis 52 (Suppl 4): S346-S350, 2011.

18. Park JH, Wee JH, Choi SP and Oh SH: The value of procalcitonin level in community-acquired pneumonia in the ED. Am J Emerg Med 30: 1248-1254, 2012.

19. Clyne B and Olshaker JS: The C-reactive protein. J Emerg Med 17: 1019-1025, 1999.
20. O'Donnell RA, Peebles C, Ward JA, Daraker A, Angco G, Broberg P, Pierrou S, Lund J, Holgate ST, Davies DE, et al: Relationship between peripheral airway dysfunction, airway obstruction, and neutrophilic inflammation in COPD. Thorax 59: $837-842,2004$

This work is licensed under a Creative Commons Attribution-NonCommercial-NoDerivatives 4.0 International (CC BY-NC-ND 4.0) License. 